\title{
Sensitivity of high-resolution ultrasonography in clinically diagnosed carpal tunnel syndrome patients with hand pain and normal nerve conduction studies
}

This article was published in the following Dove Press journal:

Journal of Pain Research

\author{
Reza Salman Roghani ${ }^{1,2}$ \\ Mohammad Taghi Holisaz ${ }^{2}$ \\ Ali Asghar Sahami Norouzi ${ }^{2}$ \\ Ahmad Delbari ${ }^{3}$ \\ Faeze Gohari ${ }^{4}$ \\ Johan Lokk' \\ Andrea J Boon ${ }^{5,6}$ \\ 'Department of Neurobiology, Care \\ Sciences and Society, Karolinska \\ Institute, Stockholm, Sweden; \\ ${ }^{2}$ Physical Medicine and Rehabilitation \\ Research Center, Shahid Beheshti \\ University of Medical Sciences, Tehran, \\ Iran; ${ }^{3}$ Research Center on Aging, \\ University of Social Welfare and \\ Rehabilitation, Tehran, Iran; ${ }^{4}$ Student \\ Research Committee, Faculty of \\ Medicine, Iran University of Medical \\ Sciences, Tehran, Iran; ${ }^{5}$ Department of \\ Physical Medicine and Rehabilitation, \\ Mayo Clinic, Rochester, MN, USA; \\ ${ }^{6}$ Department of Neurology, Mayo \\ Clinic, Rochester, MN, USA
}

Correspondence: Andrea J Boon Department of Physical Medicine and Rehabilitation, Mayo Clinic, 200 First Street, S.W. Rochester, MN 55905, USA Email boon.andrea@mayo.edu
Background: Suspecting carpal tunnel syndrome (CTS) in patients with hand pain is usual. Considering the variable rate of false-negative results in nerve conduction study (NCS), as a frequent reference confirmatory standard test, we aimed to evaluate the diagnostic accuracy of neuromuscular ultrasound in patients with clinical evidence of CTS and normal NCS.

Methods: It was a diagnostic accuracy study conducted in the outpatient clinic of Rofaydeh Hospital, Tehran, Iran, between July 2012 and December 2016; it recruited clinically diagnosed CTS patients and a control group. All participants underwent comprehensive clinical examination, NCS, and high-resolution ultrasonography of the median nerve.

Results: Two hundred and fifty patients with clinical evidence of CTS met the inclusion criteria, of whom 103 (27.1\%) had normal NCS and underwent an ultrasound examination. A cutoff point of $9.4 \mathrm{~mm}^{2}$ (mean +2 standard deviation) for median nerve cross-sectional area at the carpal tunnel inlet from the control group was set to detect $73 \%$ abnormality in the case group. Conclusion: Ultrasonography had a sensitivity rate of $73 \%$ in patients with clinical CTS and negative NCS, increasing the overall diagnostic sensitivity for clinically suspected CTS in the electrodiagnostic lab setting to $92 \%$. The study highlights the complementary role of ultrasonography in diagnosing CTS in conjunction with NCS.

Keywords: hand pain, sensitivity, carpal tunnel syndrome, nerve conduction, ultrasonography, false-negative reaction, diagnosis, complementary

\section{Background}

Carpal tunnel syndrome (CTS) is one of the most common entrapment neuropathies affecting as much as $5 \%$ of the adult population. ${ }^{1,2}$ CTS diagnosis is often suspected when typical symptoms including pain, dysesthesia in the median nerve territory, and loss of dexterity are present. ${ }^{3}$ While no real diagnostic gold standard exists, there is a strong consensus on the clinical findings necessary to diagnose CTS. These are characteristic history, physical examination findings of sensory or motor impairment in the median nerve distribution, and positive provocative tests. ${ }^{4}$ To prevent permanent devastating sequel, early diagnosis seems to be essential. ${ }^{5}$ Electrodiagnostic testing (EDX) most often confirms the diagnosis but limitations include patient discomfort and a variable rate of false-negative results. ${ }^{6,7}$ Moreover, EDX provides physiologic information regarding slowing of nerve conduction and axon loss but does not give anatomic details reflecting the underlying cause. ${ }^{7}$ Considering abovementioned shortcomings 
of EDX in CTS patients, there has been a growing trend in search of other diagnostic methods. ${ }^{8-10}$ The objective of the current study was to evaluate the diagnostic accuracy of neuromuscular ultrasound in patients with clinical evidence of CTS and normal nerve conduction study (NCS).

\section{Materials and methods Subjects}

It was a prospective diagnostic accuracy study of patients with clinically suspected CTS who were referred to our academic EDX Lab between July 2012 and December 2015 at the University Rofaydeh Rehabilitation Hospital, Tehran, Iran, using convenience sampling. We recruited the patients with clinically suspected CTS via word of mouth through their referring physician (from Orthopedic Surgery, Neurology, Neurosurgery, Physiatry, Internal Medicine); 41\% were internal referrals, and 59\% were referred from outside clinics. The clinical diagnosis of CTS was established based on the American Academy of Orthopedic Surgeons clinical practice guideline recommendations. ${ }^{10,11}$ We also included control subjects with the same age and sex distribution without clinical evidence of CTS nor any other upper limb complaint among the hospital staff, medical students, and patients' relatives. The remuneration offered to control subjects was same as that offered to patients with clinically suspected CTS.

All patients and controls underwent clinical examination, NCS, and sonography. Additionally, patients with suspected CTS also underwent needle electromyography (EMG). Demographic data including age, sex, weight, height, and body mass index (BMI) were extracted prospectively.

We excluded patients with a previous history of wrist or hand trauma and surgery, the presence of other neurologic disorders such as polyneuropathy, anatomical or structural misalignment in upper limb, and other lesions within the carpal tunnel. ${ }^{12}$

Potential subjects were offered a nominal payment and free EDX testing as an incentive for participation. The Ethics Committee of the University of Social Welfare and Rehabilitation approved the study protocol. We also adhered to the Declaration of Helsinki (Sixth Revision - 2008) and obtained written informed consent from every patient.

\section{Sampling and sample size}

The primary outcome was the median nerve cross-sectional area (CSA) at the carpal tunnel inlet level, which is the most sensitive and specific parameter in detecting CTS. ${ }^{9}$ Based on the sample size formula for estimating a population mean, ${ }^{25}$ given the absolute precision of 0.1 and a standard deviation (SD) of 0.2 considering the mean, median nerve inlet CSA in the first 20 control subjects, a sample size of at least 96 cases in each group was needed.

\section{Clinical diagnosis}

A single physician examined all patients and controls based on the American Academy of Orthopedic Surgeons clinical practice guideline recommendations. ${ }^{10,11}$ The examiner included detailed history taking and personal characteristics, pace activities, and comorbidities. He also performed standard sensory examination, manual muscle testing of the upper extremity, and provocative tests such as Phalen's test and compression test and discriminatory tests such as Spurling test for alternative diagnoses. A translated and valid version of Boston pain questionnaire was the final step to confirm the diagnosis clinically.

To be as specific as possible in clinical diagnosis, only patients with positive subjective and objective and Boston questionnaire results were considered as having suspected CTS.

\section{Electrodiagnostic studies}

Just one physiatrist, who was unaware of the subjects' clinical status, with 25 years of EDX experience conducted the EDX studies of each arm. He used A Neuropack EMG machine (S1, MEB-9400K; Japan) for all studies. The skin and room temperature were more than $32^{\circ} \mathrm{C}$ and at $25^{\circ} \mathrm{C}$, respectively.

He performed EDX according to the American Association of Neuromuscular and Electrodiagnostic Medicine (AANEM) standard criteria for suspected CTS. ${ }^{7}$ All subjects underwent median antidromic sensory NCS across the wrist with a conduction distance of 13-14 cm. If it was normal, the ulnar sensory, mixed palmar orthodromic median and ulnar NCS were performed to evaluate the absolute or relative slowing of the median orthodromic latency. ${ }^{7} \mathrm{Bi}$-hump sensory response technique from 4th finger, with inter hump difference more than $3 \mathrm{msec}$, was also considered to detect milder forms of CTS. ${ }^{7}$ Subjects with suspected CTS also underwent median, ulnar, and radial motor NCS, medial and lateral antebrachial cutaneous sensory NCS, and needle EMG of the upper limb to exclude any differential diagnosis or existed comorbidities, eg, cervical radiculopathy or brachial plexopathy. ${ }^{13}$ Reference values established by Dumitru were used. ${ }^{22}$ We applied all the abovementioned examinations for control subjects except needle examination. 


\section{Sonography}

High-resolution ultrasonography of the carpal tunnel was carried out by a single musculoskeletal sonographer with 3 years of experience who did not know the clinical status and NCS results. He performed scans according to the European guidelines for musculoskeletal sonography of the median nerve. ${ }^{11-14}$

Subjects were seated in front of the sonographer during the scan, with the forearm in relaxed supination, fingers in the semi-flexed position, and the elbow flexed to approximately 60 degrees. We used a My-lab 25 (Esaote, Genoa, Italy) ultrasound machine with a $10-18 \mathrm{MHz}$ linear array transducer. The frequency, depth, gain, and focal zone were kept constant for all studies. Care was taken to rest the probe gently on the skin/gel interface. The sonographer positioned the probe at the level of the distal wrist crease in a transverse plane. CSA of median nerve was measured at the carpal tunnel inlet (level of the pisiform and scaphoid) by tracing just inside the hyperechoic margin with integrated measurement software of abovementioned sonography machine, ${ }^{15}$ as demonstrated in Figure 1; in the case of a bifid nerve at the inlet level, two CSAs were combined. ${ }^{16}$ Three measurements were made, and their mean was recorded as the median nerve CSA. Intra-rater measurement reliability was tested in study subjects by having the sonographer capture and save two unmarked images. The same person measured the median nerve CSA on each of the saved images, three times in a week.

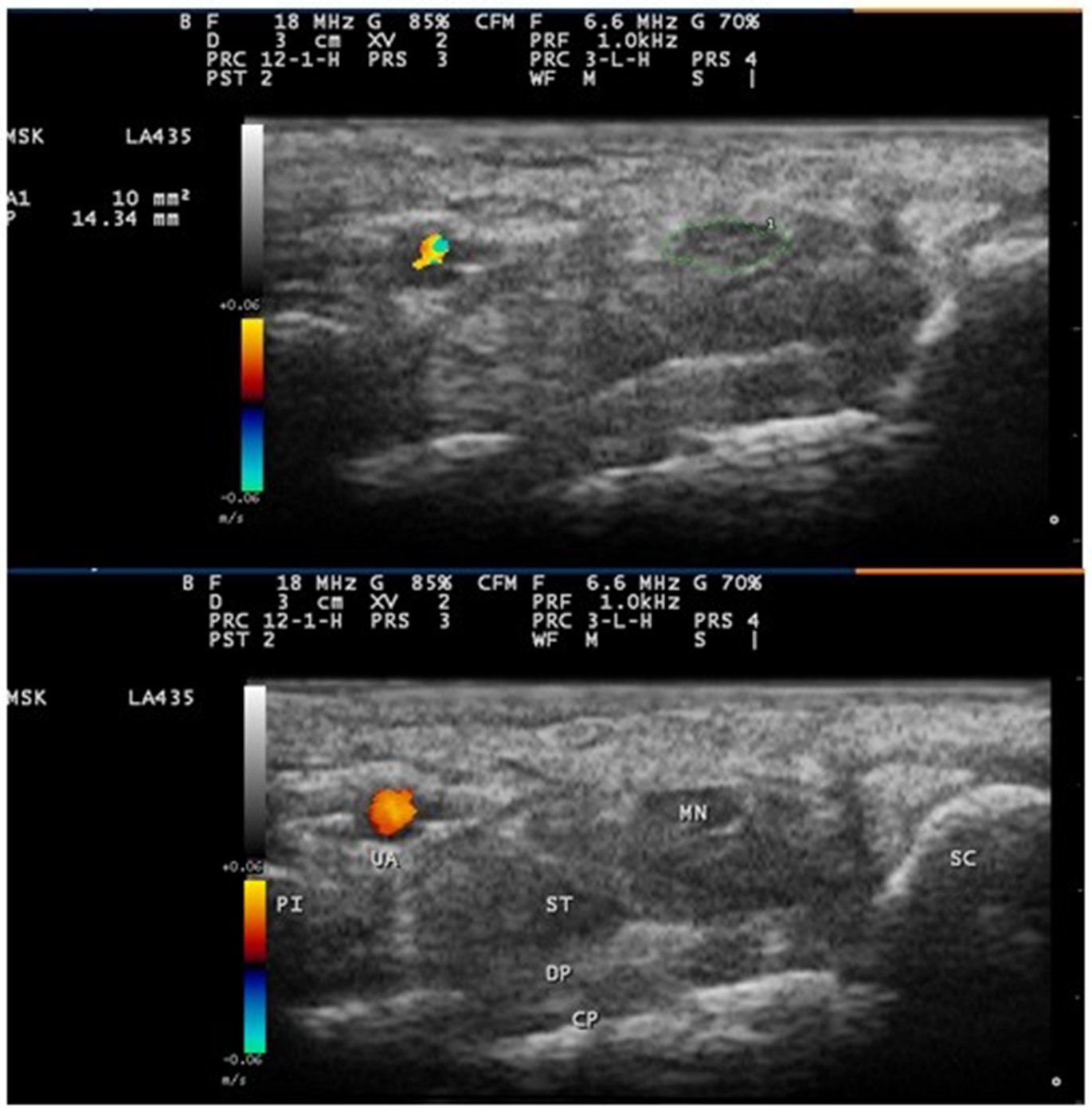

Figure I Ultrasound image of the median nerve at the carpal tunnel inlet. The image shows the median nerve outlined using a free trace method inside the hyperechoic rim of the nerve.

Abbreviations: CP, carpal bones (lunate); DP, deep tendons (flexor digitorum profundus); MN, median nerve; PI, pisiform bone; SC, scaphoid bone; ST, superficial tendons (flexor digitorum superficialis); UA, ulnar artery. 


\section{Statistical analysis}

SPSS version 19 (SPSS Inc., Chicago, IL, USA) was used for statistical analysis. Parametric variables were depicted as the mean $\pm \mathrm{SD}$, with the remainder presented as frequencies and percentages. Distribution of data was tested with the Kolmogorov-Smirnov test. An $\alpha$-value $<0.05$ was considered significant. Independent $t$-test was used to compare quantitative variables between CTS patients and the reference group of the study. Dependability coefficient (phi) was used for intra-rater reliability.

\section{Results}

During the study period, 2830 patients were referred to our EMG lab, of whom 1923 patients had upper limb complaints. Based on abovementioned clinical examination, 1243 patients were diagnosed CTS clinically. Among them, 250 agreed to participate in the study. Of these 250 volunteers, 130 patients had bilateral CTS and 120 patients had unilateral CTS, thus totally to 380 affected wrists. NCSs confirmed
CTS diagnosis in 269 (73\%) wrists, and was normal in the rest $(27 \%)$. In the final item by item NCS data check, we found prolonged median sensory distal latency $(>3.7 \mathrm{msec})$ in 5 cases and a slowed median conduction velocity at the forearm level $(<50 \mathrm{~m} / \mathrm{sec})$ in other 3 cases. These eight wrists were initially included due to some shortcomings in report generation or data entry and therefore were excluded from data analysis, leaving 103 wrists for the final analysis. The control group was made up of 108 wrists without CTS symptoms. Nine were found to have abnormal median NCS and therefore were excluded, leaving 99 wrists for the final analysis. The recruitment flow diagram is presented in Figure 2.

BMI and median CSA had significant difference between case and control groups' demographic data (Table 1). The correlation between CSA and BMI was not detected neither in CTS patients $(P$-value $=0.57$, Pearson rho $=0.55)$ nor in the control group $(P$-value $=0.29$, Spearman $r h o=-0.107)$.

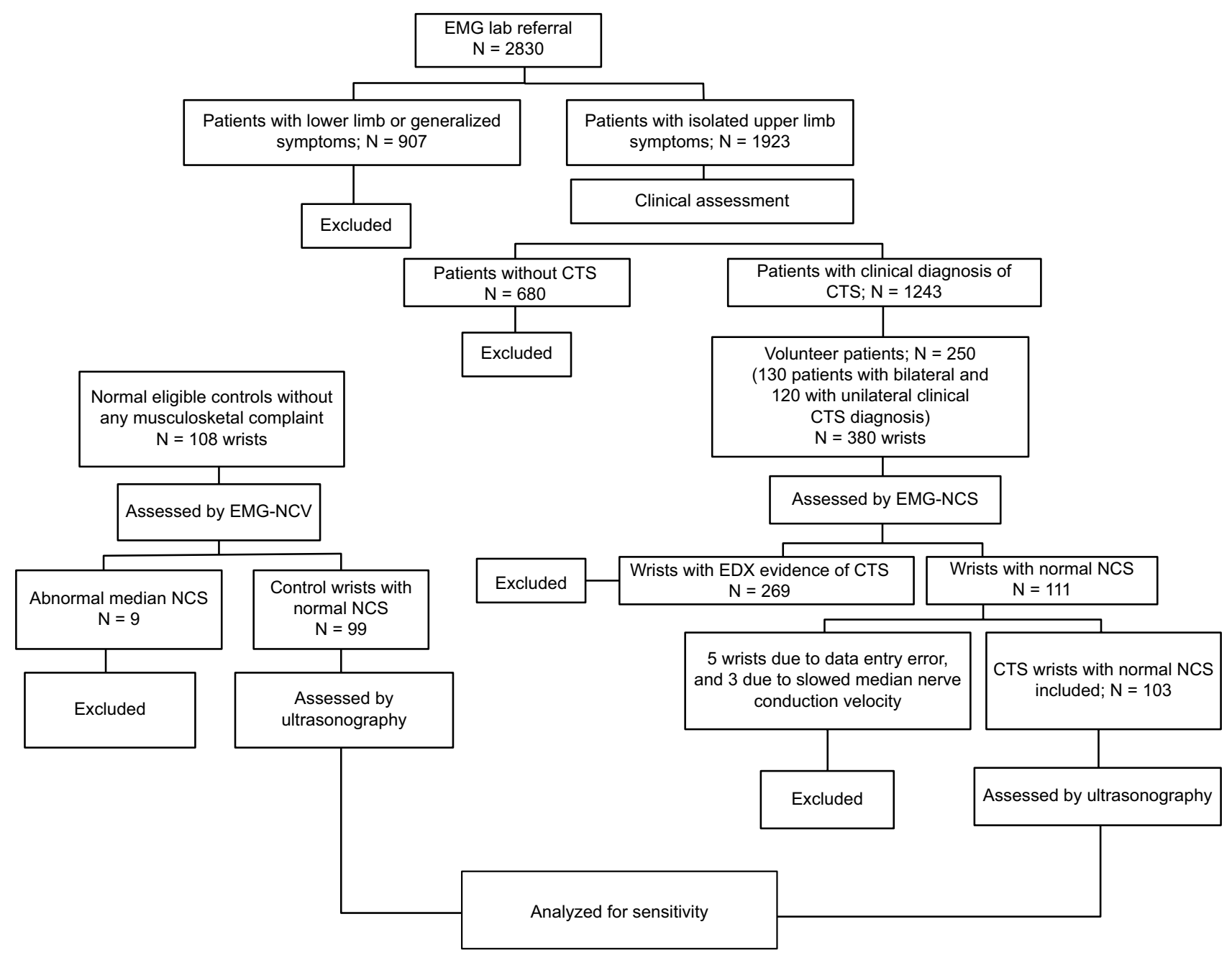

Figure 2 Study design flowchart.

Abbreviations: CTS, carpal tunnel syndrome; EDX, electrodiagnostic testing; EMG, electromyography; NCS, nerve conduction study. 
Table I Demographic data of the patients and control subjects

\begin{tabular}{llll}
\hline Variable & $\begin{array}{l}\text { Controls } \\
(\mathbf{n = 9 9 )}\end{array}$ & $\begin{array}{l}\text { Patients } \\
(\mathbf{n = 1 0 3 )}\end{array}$ & P-value \\
\hline Age, mean \pm SD & $40.7 \pm 11.8$ & $40 \pm 10.8$ & 0.117 \\
Sex (female/male) & $53 / 46$ & $59 / 44$ & 0.798 \\
Height (cm), mean \pm SD & $165.7 \pm 7.4$ & $165.3 \pm 8.7$ & 0.687 \\
Weight (kg), mean \pm SD & $68.4 \pm 5.9$ & $71.4 \pm 9.9$ & 0.108 \\
BMI, mean \pm SD & $24.9 \pm 2$ & $26.2 \pm 3.9$ & $0.003^{*}$ \\
Median nerve CSA at inlet & $7 \pm 1$ & $11.6 \pm 3.1$ & $0.00 I^{*}$ \\
level mm², mean \pm SD & & &
\end{tabular}

Note: *Statistically significant.

Abbreviations: BMI, body mass index; CSA, cross-sectional area; SD, standard deviation.

Table 2 Median nerve CSA at the carpal tunnel inlet in healthy subjects (controls) and in patients with clinically diagnosed carpal tunnel syndrome (cases)

\begin{tabular}{llll}
\hline Values & Controls & $\begin{array}{l}\text { Cases } \\
(\text { CSA* }<9.4)\end{array}$ & $\begin{array}{l}\text { Cases } \\
(\text { CSA } \geq 9.4)\end{array}$ \\
\hline $\mathrm{N}$ & 99 & 28 & 75 \\
Minimum CSA & 5.3 & 7.1 & 9.8 \\
Maximum CSA & 11.2 & 9 & 19.7 \\
Mean CSA (SD) & $7.1(1.15)$ & $8.1(0.64)$ & $13.3(2.22)$ \\
Mean CSA + 2SD & 9.4 & 9.2 & 17.7 \\
\hline
\end{tabular}

Note: *Cases with normal CSA

Abbreviations: CSA, cross-sectional area; SD, standard deviation.

The sonographic findings of the median nerve are shown in Table 2. From the control wrists' values, we extracted a cutoff level of $9.4 \mathrm{~mm}^{2}$, applying 2SD above the mean CSA as the threshold in CTS diagnosis. By this measure, 75 wrists (73\%) were diagnosed with CTS, given the sensitivity of $73 \%$ for high-resolution sonography in clinically diagnosed CTS with normal NCSs.

Additionally, out of 380 total wrists, with clinically confirmed CTS, 277 had CTS in EDX. When both methods were considered in a complementary manner, we achieved $92.6 \%$ sensitivity. Intra-rater reliability, as described in the Materials and methods section, gave a Phi equal to 0.937 .

\section{Discussion}

The current study demonstrated that ultrasound had sensitivity of $73 \%$ in clinically diagnosed CTS wrists with normal NCS, whereas the pooled sensitivity of ultrasound and NCS in clinically diagnosed CTS wrists was as high as $92.6 \%$.

Clinical findings are the cornerstone for the diagnosis of CTS, and confirmation by EDX findings is widely used as the most accurate diagnosis. ${ }^{3}$ However, shortcoming in EDX standard references value and electrophysiologic changes delay in acute CTS may be responsible for false-negative EDX results. On the other hand, relatively invasive nature of EDX has led physicians to search for another diagnostic methods. ${ }^{3,4,7,8}$
Ultrasonography is an alternative confirmatory method for the diagnosis of CTS, according to recently published meta-analyses ${ }^{17}$ restricting the role of electrodiagnosis to people with advanced axonal loss and suspected for differential diagnosis. ${ }^{18}$ We chose to measure CSA of the median nerve at the carpal tunnel inlet, as this has been found to be the most sensitive and specific sonographic variable, based on a literature review of published abstracts between 2000 and 2017,, $, 18,19$ as well as a recent meta-analysis published by the AANEM. ${ }^{20}$ However, no exact normal ranges for median nerve CSA and cutoff point for detecting CTS have been developed, mainly due to variations in equipments, measurement techniques, patients' characteristics and so the variable location of maximum CSA or median nerve swelling, etc. In a recent meta-analysis, the most frequent CSA cutoff point for detecting CTS was between 8.5 and $10 \mathrm{~mm}^{2}$ at the level of the pisiform. ${ }^{18}$

Koyuncuoglu et al found abnormal median nerve CSA in $30 \%$ of 59 wrists with clinical diagnosis of CTS patients and normal NCS, using a median nerve CSA $>10.5 \mathrm{~mm}^{2}$ at the carpal tunnel inlet as a diagnostic cutoff. ${ }^{20}$ Another study detected a sensitivity of $48.6 \%$ with almost the same population and sonographic technique. ${ }^{21}$ We found a significantly higher sensitivity for sonography in this setting, which may in part be due to our larger sample size, lower CSA cutoff, lower sensitivity of our NCS in diagnosing CTS, or a combination of the abovementioned criteria. Although we did follow the AANEM practice parameter guideline in choosing the specific NCS values to diagnose CTS, in conjunction with standard reference values, ${ }^{7,22}$ the relatively low sensitivity for detecting CTS with NCS in our lab suggests we may need to establish NCS normal values specific for us.

The cutoff point (mean $+2 \mathrm{SD}$ ) for the median nerve CSA at the level of pisiform in our control group was $9.36 \mathrm{~mm}^{2}$, which correlates well with other standard high-quality studies, ${ }^{3,6}$ and was rounded up to $9.4 \mathrm{~mm}^{2}$. Many clinical tests fall short of their ideal by choosing an incorrect optimal cutoff value. The methodology of cutoff value determination is multiple, including $95 \%$ confidence interval for mean (mean+2SD), logistic regression, receiver operating characteristics curves, and discriminant analysis. ${ }^{23} \mathrm{~A}$ right selection of diagnostic test and cutoff value determination method can provide an optimal cutoff with optimal sensitivity and specificity. Mean $+2 \mathrm{SD}$ as a conventional crude method of obtaining cutoff values for a diagnostic test may be subjected to some drawbacks. So, the upper limit of healthy persons may not coincide with the lower limit of patients. Sometimes a gap between the two and sometimes an overlap may exist. Moreover, such variability in the methodology of cutoff point 
determination may also be a potential reason in observed wide ranges of sensitivity.

Inter-rater and intra-rater reliabilities are always of concern in operator-dependent tests such as ultrasound and, ideally, should be addressed in research studies. Conversely, the intra-rater and inter-rater reliabilities of various median nerve measurements about CTS are usually ignored. ${ }^{24}$ These variabilities justify the discrepancies in sensitivity of ultrasonography in diagnosing CTS patients with normal NCS. Our study protocol only used one sonographer (unaware of the patient's clinical status), and our intra-rater reliability rate was 0.937 dependability coefficient, demonstrating excellent repeatability.

As a limitation we analyzed both wrists from the same patient if they had bilateral symptoms, because of funding constraints, to reach adequate sample size based on our power analysis. Another limitation was that we excluded patients with other diagnoses, which could mimic CTS, based on their clinical examination and EDX testing, eg, proximal median neuropathy or C6 radiculopathy; this can by itself reduce the generalizability of our finding.

We were not attempting to evaluate the sensitivity of ultrasound in diagnosing CTS in isolation but in conjunction with NCS. The strengths of our study were its prospective design, the inclusion of patients who were newly diagnosed, and reporting the intra-rater reliability.

Although our objective was to determine the sensitivity and specificity of ultrasound in diagnosing CTS in clinically suspected CTS with normal NCS, it is important to remember that these tests should be used judiciously, only when clinically indicated, in conjunction with a detailed history and physical examination findings.

\section{Conclusion}

The present study highlights the complementary role of ultrasound in diagnosing CTS jointly with EDX, particularly in patients with clinical diagnosis of CTS and negative NCS. Sonography was $73 \%$ sensitive in patients with CTS and negative NCS, increasing the overall diagnostic sensitivity for clinically suspected CTS in our lab setting to $92 \%$ in conjunction with EDX. The authors recommend using diagnostic ultrasound in EDX labs, especially for patients with clinical findings suggestive of CTS but with normal NCS.

\section{Acknowledgments}

The study was supported by a grant from the Physical Medicine and Rehabilitation Research Center, Shahid
Beheshti University of Medical Sciences, Tehran, Iran. Also, we would like to thank all the patients for their collaborations.

To the best of our knowledge, this manuscript has not been considered for publication elsewhere, except for its primary results" oral presentation in "9th World Congress of the International Society of Physical and Rehabilitation Medicine” June 2015.

\section{Author contributions}

All authors contributed toward study design and conception, data analysis, drafting and critically revising the paper, gave final approval of the version to be published, and agree to be accountable for all aspects of the work.

\section{Disclosure}

The authors report no conflicts of interest in this work.

\section{References}

1. Atroshi I, Gummesson C, Johnsson R, Ornstein E, Ranstam J, Rosén I. Prevalence of carpal tunnel syndrome in a general population. JAMA. 1999;282(2):153-158.

2. Ibrahim I, Khan WS, Goddard N, Smitham P. Carpal tunnel syndrome: a review of the recent literature. Open Orthop J. 2012;6:69-76.

3. Padua L, Coraci D, Erra C, et al. Carpal tunnel syndrome: clinical features, diagnosis, and management. Lancet Neurol. 2016;15(12):1273-1284.

4. El Miedany YM, Aty SA, Ashour S. Ultrasonography versus nerve conduction study in patients with carpal tunnel syndrome: substantive or complementary tests? Rheumatology (Oxford). 2004;43(7): 887-895.

5. Wong SM, Griffith JF, Hui AC, Lo SK, Fu M, Wong KS. Carpal tunnel syndrome: diagnostic usefulness of sonography. Radiology. 2004;232(1):93-99.

6. Kolovos S, Tsiotas D. Ultrasonographic diagnosis of carpal tunnel syndrome: introducing a new approach. Eur J Orthop Surg Traumatol. 2016;26(2):167-175

7. Jablecki CK, Andary MT, Floeter MK, et al; American Association of Electrodiagnostic Medicine; American Academy of Neurology; American Academy of Physical Medicine and Rehabilitation. Practice parameter: electrodiagnostic studies in carpal tunnel syndrome Report of the American Association of Electrodiagnostic Medicine, American Academy of Neurology, and the American Academy of Physical Medicine and Rehabilitation. Neurology. 2002;58(11):1589-1592.

8. Jarvik JG, Yuen E, Haynor DR, et al. MR nerve imaging in a prospective cohort of patients with suspected carpal tunnel syndrome. Neurology. 2002;58(11):1597-1602.

9. Cartwright MS, Hobson-Webb LD, Boon AJ, et al; American Association of Neuromuscular and Electrodiagnostic Medicine. Evidence-based guideline: neuromuscular ultrasound for the diagnosis of carpal tunnel syndrome. Muscle Nerve. 2012;46(2):287-293.

10. Sucher BM, Schreiber AL. Carpal tunnel syndrome diagnosis. Phys Med Rehabil Clin N Am. 2014;25(2):229-247.

11. Klauser AS, Tagliafico A, Allen GM, et al. Clinical indications for musculoskeletal ultrasound: a Delphi-based consensus paper of the European Society of Musculoskeletal Radiology. Eur Radiol. 2012;22(5): $1140-1148$ 
12. Klauser AS, Halpern EJ, De Zordo T, et al. Carpal tunnel syndrome assessment with US: value of additional cross-sectional area measurements of the median nerve in patients versus healthy volunteers. Radiology. 2009;250(1):171-177.

13. Cannella AC, Kissin EY, Torralba KD, Higgs JB, Kaeley GS. Evolution of musculoskeletal ultrasound in the United States: implementation and practice in rheumatology. Arthritis Care Res (Hoboken). 2014;66(1):7-13.

14. Backhaus M, Burmester GR, Gerber T, et al; Working Group for Musculoskeletal Ultrasound in the EULAR Standing Committee on International Clinical Studies including Therapeutic Trials. Guidelines for musculoskeletal ultrasound in rheumatology. Ann Rheum Dis. 2001;60(7):641-649.

15. Klauser AS, Abd Ellah MM, Halpern EJ, et al. Sonographic crosssectional area measurement in carpal tunnel syndrome patients: can delta and ratio calculations predict severity compared to nerve conduction studies? Eur Radiol. 2015;25(8):2419-2427.

16. Martinoli C. Musculoskeletal ultrasound: technical guidelines. Insights Imaging. 2010;1(3):99-141.

17. Fowler JR, Gaughan JP, Ilyas AM. The sensitivity and specificity of ultrasound for the diagnosis of carpal tunnel syndrome: a meta-analysis. Clin Orthop Relat Res. 2011;469(4):1089-1094.

18. Torres-Costoso A, Martínez-Vizcaíno V, Álvarez-Bueno C, FerriMorales A, Cavero-Redondo I. Accuracy of ultrasonography for the diagnosis of carpal tunnel syndrome: a systematic review and metaanalysis. Arch Phys Med Rehabil. 2018;99(4):758-765.e10.
19. Pan TJ, White RJ, Zhang C, Hagberg WC, Imbriglia JE, Fowler JR Baseline characteristics of the median nerve on ultrasound examination. Hand (NY). 2016;11(3):353-356.

20. Koyuncuoglu HR, Kutluhan S, Yesildag A, Oyar O, Guler K, Ozden A. The value of ultrasonographic measurement in carpal tunnel syndrome in patients with negative electrodiagnostic tests. Eur J Radiol. 2005;56(3):365-369.

21. Al-Hashel JY, Rashad HM, Nouh MR, et al. Sonography in carpal tunnel syndrome with normal nerve conduction studies. Muscle Nerve. 2015;51(4):592-597.

22. Dumitru D, Zawarts MJ. Focal peripheral neuropathies . In: Dumitru D, Amato AA, Zawarts MJ, editors. Electrodiagnostic Medicine. Philadelphia, PA: Hanley \& Belfus; 2002:1060.

23. Sharma B, Jain R. Right choice of a method for determination of cut-off values: a statistical tool for a diagnostic test. Asian J Med Sci. 2014;5(3):30-34.

24. Impink BG, Gagnon D, Collinger JL, Boninger ML. Repeatability of ultrasonographic median nerve measures. Muscle Nerve. 2010;41(6):767-773.

25. Binu VS, Mayya SS, Dhar M. Some basic aspects of statistical methods and sample size determination in health science research. Ayu. 2014;35(2):119-123.

\section{Journal of Pain Research}

\section{Publish your work in this journal}

The Journal of Pain Research is an international, peer reviewed, open access, online journal that welcomes laboratory and clinical findings in the fields of pain research and the prevention and management of pain. Original research, reviews, symposium reports, hypothesis formation and commentaries are all considered for publication.

\section{Dovepress}

The manuscript management system is completely online and includes a very quick and fair peer-review system, which is all easy to use. Visit http://www.dovepress.com/testimonials.php to read real quotes from published authors. 